\title{
PACIENTES CON ENFERMEDAD CRÓNICA TERMINAL EN EL SERVICIO DE EMERGENCIA DE ADULTOS DE UN HOSPITAL TERCIARIO
}

\author{
PATIENTS WITH TERMINAL CHRONIC DISEASE IN THE ADULT EMERGENCY \\ SERVICE OF A TERTIARY HOSPITAL
}

\author{
José Amado-Tineo 1,3,a,e, Waldo Taype-Huamaní1,b, Rolando Vasquez-Alva',3,a,d, \\ Fiorella Siccha-Del Campoc, Teodoro Oscanoa-Espinoza 2,4,a,e
}

\begin{abstract}
RESUMEN
Introducción: Pacientes con enfermedad crónica terminal acuden con frecuencia a emergencia de hospitales de referencia. Objetivo: Determinar la frecuencia de enfermedad crónica terminal en pacientes admitidos a emergencia, identificar procedimientos invasivos y comparar oncológicos con no oncológicos. Métodos: Estudio de corte transversal, en mayores de 18 años admitidos a emergencia de un hospital terciario, noviembre 2017. Se consideró enfermedad terminal al cáncer en estadio clínico IV, deterioro cognitivo severo o dependencia funcional total más diagnóstico de demencia, secuela neurológica, dependencia de oxígeno, índice de filtración glomerular $<30 \mathrm{ml} / \mathrm{min}$, cirrosis hepática Child C o fracción de eyección ventricular izquierda $<20 \%$. Análisis estadístico con U de Mann-Whitney y Chi cuadrado. Resultados: El 10,7\% (172 de 1604 admisiones) de los pacientes admitidos presentaron criterios de enfermedad crónica terminal, de causa oncológica $21,5 \%(37 / 172)$ y no oncológica $78,5 \%$ (135/172). En oncológicos la mediana de edad fue 70 años y sexo femenino $59,5 \%$, mientras que en no oncológicos 84 años y $57 \%$ de mujeres ( $p<0,01$ y 0,79 respectivamente). El $41 \%$ $(71 / 172)$ ingresó por unidad de shock trauma, con mayor frecuencia por somnolencia y disnea. Se identificó procedimiento invasivo probablemente innecesarios en $21,5 \%$ (37/172 pacientes), intubación orotraqueal y catéter venoso central para inotrópicos fueron los más frecuentes. La estancia promedio en emergencia fue ocho días, falleciendo en emergencia $32 \%$ de oncológicos y $24 \%$ de no oncológicos $(p=0,43)$. Conclusión: El $10 \%$ de los pacientes admitidos a emergencia de un hospital terciario presenta enfermedad crónica terminal, con mayor frecuencia no oncológica, realizándose procedimientos probablemente innecesarios en la quinta parte de estos.
\end{abstract}

Palabras clave: Enfermedad crónica terminal; Admisión; Servicio de emergencia. (fuente: DeCS BIREME)

\begin{abstract}
Interoduction: Patients with chronic terminal disease frequently come to emergency department of reference hospitals. Objective: To determine frequency of chronic terminal disease in patients admitted to emergency department, identify invasive procedures and compare oncological with non-oncological patients. Methods: Cross-sectional study in patients older than 18 years old, admitted to emergency department of a tertiary hospital, November 2017. Criteria for terminal disease: Clinical stage IV cancer, severe cognitive deterioration or total functional dependence plus diagnosis of dementia, neurological sequelae, dependence of oxygen, glomerular filtration rate $<30 \mathrm{ml} / \mathrm{min}$, Child C cirrhosis, or left ventricular ejection fraction <20\%. Statistical analysis with Mann-Whitney $U$ and Chi square tests. Results: $10.7 \%$ (172 of 1604 admissions) of admitted patients presented criteria of chronic terminal disease, with oncological cause $21.5 \%$ (37/172) and non-oncological $78.5 \%$ (135/172). In oncological patients, the median age was 70 years and was female $59.5 \%$, while in non-oncological 84 years and $57 \%$ of women ( $p<0.01$ and 0.79 , respectively). $41 \%(71 / 172)$ were admitted by shock-trauma unit, more frequently due to drowsiness and dyspnea. Invasive procedures, probably unnecessary, were identified in $21.5 \%$ (37/172 patients), orotracheal intubation and central venous catheter for inotropic were the most frequent. The average stay in emergency department was eight days, with $32 \%$ of oncological deaths and $24 \%$ of non-oncological deaths in emergency department $(p=0.43)$. Conclusion: $10 \%$ of patients admitted to the emergency department of a tertiary hospital have chronic terminal disease, with a greater frequency of non-oncology, probably performing unnecessary procedures in one fifth of these.
\end{abstract}

Key words: Chronic terminal disease; Admission; Emergency department. (source: MeSH NLM)

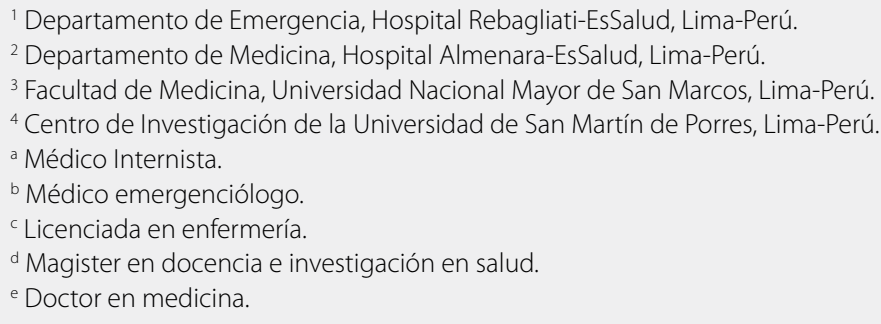

Citar como: Mosé Amado-Tineo, Waldo Taype-Huamaní, Rolando Vasquez-Alva, Fiorella Siccha-Del Campo, Teodoro Oscanoa-Espinoza. Pacientes con enfermedad crónica terminal en el servicio de emergencia de adultos de un hospital terciario. Rev. Fac. Med. Hum. Julio 2019; 19(3):27-34. DOI 10.25176/RFMH.v19i3.2166 


\section{INTRODUCCIÓN}

El servicio de emergencia (urgencia) de un hospital está diseñado y equipado para brindar atención inmediata a pacientes con problemas de salud que pongan en riesgo su vida o produzcan secuelas invalidantes. El principal objetivo en este servicio es salvar la vida, estabilizando al paciente para su posterior tratamiento definitivo'.

En las últimas décadas, el aumento de la demanda de atención, debido a incremento poblacional (por avance tecnológico, transición epidemiológica y migración), ha producido saturación de estos servicios, principalmente en los grandes hospitales de referencia, predominando pacientes con enfermedades crónicas, complejas y de edad avanzada, desviando esfuerzos diagnósticos y terapéuticos ${ }^{2,3}$.

Internacionalmente se ha definido criterios para la fase final o terminal de enfermedades crónicas (oncológicas y no oncológicas), donde se han producido daños irreversibles, con múltiple sintomatología, pronóstico de vida limitado y gran impacto emocional para el paciente, la familia y el personal de salud. La OMS recomienda cuidados paliativos en esta fase de enfermedad y organismos internacionales han especificado que ciertos procedimientos invasivos son innecesarios, más que ayudar al paciente pueden prolongar su sufrimiento y el de la familia ${ }^{4-6}$.

La frecuencia de enfermedad en fase terminal en servicios de emergencia es variable y subestimada6; estudios realizados en Norteamérica, Europa y Asia han demostrado que el empleo de cuidados paliativos en pacientes con enfermedades terminales (institucionalizados y seguimiento domiciliario), disminuye admisiones hospitalarias, ingresos a cuidados intensivos y frecuentación a urgencias; sin diferencia significativa en el tiempo de sobrevida de estos pacientes; mejorando mucho las condiciones del paciente y la familia ${ }^{7-14}$. Pero también se reporta alta frecuencia de atención de estos pacientes en emergencia, donde muchos fallecen sin recibir cuidados paliativos ${ }^{15,16}$.

En Latinoamérica y Perú son más escasos los reportes al respecto. En nuestro medio se reportó frecuencia importante de enfermedad en fase terminal (30\%) entre los pacientes readmitidos a emergencia en el último año, estancia hospitalaria prolongada, readmisión frecuente y elevada tasa de mortalidad $23,17,18$.

Esto refleja problemas en el sistema nacional de salud, con deficiencia en el enfoque de los cuidados paliativos, siendo parte de la solución mejorar la selección de pacientes y referir a estos pacientes a servicios adecuados, evitando someterlos a procedimientos innecesarios en los servicios de emergencia y brindar mejor calidad de vida a estos pacientes y su familia, sin encarnizamiento terapéutico ${ }^{11,19,20}$. Esto significa integrar cuidados paliativos a emergencia, como se viene haciendo en Europa, Oceanía y Norteamérica ${ }^{4,21,22}$.

El presente estudio tiene como objetivo determinar la frecuencia de enfermedad crónica terminal en pacientes admitidos al servicio de emergencia de adultos de un hospital terciario, identificar los tipos de enfermedad y procedimiento invasivos instaurados.

\section{MÉTODOS}

Estudio de corte transversal, en pacientes mayores de 18 años de edad admitidos a emergencia de un hospital terciario de Lima-Perú, el cual cuenta con 200 camillas de observación y reportó 164370 atenciones y 22883 admisiones al año el $2015^{2}$. La muestra fue recolectada entre 1 y 30 de noviembre de 2017.

Se consideró enfermedad oncológica terminal al diagnóstico histológico confirmado en estadio clínico IV o metástasis múltiple cerebral, hepática o pulmonar. En no oncológicos se consideró enfermedad terminal si presentaba por más de tres meses deterioro cognitivo severo (test de Pfeiffer) o dependencia funcional total (escala de Katz) más diagnóstico de demencia, secuela neurológica, falla renal con tasa de filtración glomerular $<30 \mathrm{ml} / \mathrm{min}$, enfermedad respiratoria con oxígeno domiciliario continuo, cirrosis hepática en estadio $C$ de escala Child-Pugh o falla cardíaca con fracción de eyección de ventrículo izquierdo $<20 \%^{15,16,23,24}$. Se excluyó aquellos con datos incompletos, readmitidos durante el seguimiento, fallecidos o transferidos antes de las primeras 24 horas de ser admitidos.

Para identificación inicial de pacientes se revisó los reportes médicos diarios, en los posibles participantes se revisó historia clínica y realizó entrevista/ evaluación al paciente y/o cuidador principal por una de dos enfermeras capacitadas en el tema. Los pacientes seleccionados fueron evaluados por uno de tres médicos especialistas del servicio de emergencia, capacitados en criterios de fase terminal de enfermedad. Las dudas eran resueltas en consenso con el investigador principal. Se realizó seguimiento en el sistema estadístico institucional.

Los datos fueron recolectados en una ficha prediseñada, codificados, digitado en Microsoft Excel 2010 y procesados con IBM SPSS 25.00. Para comparar los grupos oncológicos y no oncológicos, se aplicó análisis bivariado cualitativo con prueba de 
Chi cuadrado; en las variables cuantitativas se aplicó prueba de Kolmogorov-Smirnov para identificar distribución normal y U Mann-Whitney para muestras independientes en distribución no normal. Se consideró significativo $p<0,05$ e intervalo de confianza de $95 \%$.

Se obtuvo aprobación del comité de investigación institucional. Los pacientes fueron evaluados siguiendo los principios de buenas prácticas clínicas y la declaración de Helsinki, previo consentimiento informado verbal del paciente o cuidador principal.

\section{RESULTADOS}

En el periodo evaluado, el servicio de emergencia registró 14386 atenciones y 1604 admisiones. Se identificaron 172 pacientes con criterios de enfermedad crónica terminal $(21,5 \%$ de causa oncológica y 78,5\% no oncológica), determinando una prevalencia de $10,7 \%$ de pacientes con enfermedad crónica en fase terminal entre los pacientes admitidos a este servicio. Las características de pacientes según etiología se presentan en la Tabla 1, la edad varió entre 19 y 101 años. El dolor más reportado fue abdominal, lumbar y de extremidades.

Tabla 1. Características de los pacientes con enfermedad crónica en fase terminal admitidos a emergencia de un hospital terciario.

\begin{tabular}{|c|c|c|c|c|}
\hline Características & Oncológicos (37) & No Oncológicos (135) & Total (172) & $\mathbf{P}$ \\
\hline Edad en años: mediana (RI\&) & $70(16)$ & $84(17)$ & 79,5 (19) & $<0,01^{*}$ \\
\hline Sexo femenino & $22(59,5 \%)$ & 77 (57\%) & $99(58 \%)$ & $0,79 * *$ \\
\hline En servicios paliativos: & & & & $0,10^{* *}$ \\
\hline COPHOES & $2,7 \%$ & $0,7 \%$ & $1,2 \%$ & \\
\hline PADOMI & 0 & $9,6 \%$ & $7,6 \%$ & \\
\hline Lugar de procedencia: & & & & $0,04^{* *}$ \\
\hline - Domicilio & 37 (100\%) & $110(81,5 \%)$ & 147 & \\
\hline - Casa de reposo & - & $18(13,3 \%)$ & $(85,5 \%)$ & \\
\hline - Hospital o clínica & - & $7(5,2 \%)$ & $18(10,5 \%) ; 7$ (4\%) & \\
\hline Área de admisión: & & & & $0,03^{* *}$ \\
\hline - Shock trauma & $9(24,3 \%)$ & $62(45,9 \%)$ & 71 & \\
\hline - Medicina & 27 (73\%) & $61(45,2 \%)$ & $(41,3 \%)$ & \\
\hline - Cirugía/Traumatología & $1(2,7 \%)$ & $12(8,9 \%)$ & $88(51,2 \%) ; 13(7,5 \%)$ & \\
\hline Motivo de ingreso: & & & & $0,21^{* *}$ \\
\hline - Somnolencia & $4(10,8 \%)$ & $30(22,2 \%)$ & 34 & \\
\hline - Disnea & $7(19 \%)$ & $20(14,8 \%)$ & $(19,8 \%)$ & \\
\hline - Dolor & $6(16,2 \%)$ & $8(6)$ & 27 & \\
\hline • Fiebre & $2(5,4 \%)$ & $5(3,7 \%)$ & $\begin{array}{c}(15,7 \%) ; 14(8,1 \%) ; 7 \\
(4,1)\end{array}$ & \\
\hline
\end{tabular}


La figura 1, presenta los principales grupos de patologías. Las causas específicas más frecuentes en pacientes oncológicos fueron cáncer de mama, adenocarcinoma pulmonar y hepatocarcinoma. En los no oncológicos: demencia vascular, secuela de enfermedad cerebrovascular y la categoría dependiente total (sin mayores datos del motivo de postración).

\section{Oncológicos}

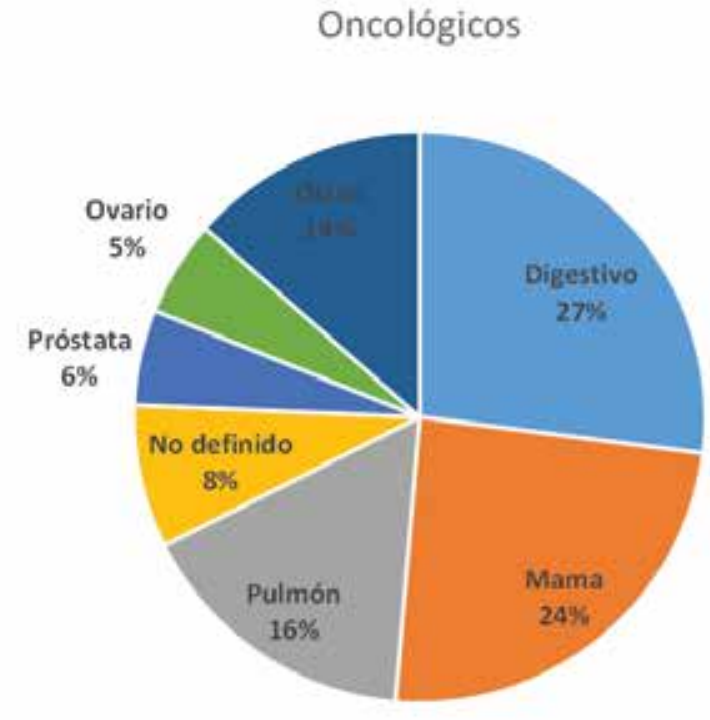

Se identificó caquexia en $11 \%$ de pacientes con enfermedad crónica en fase final oncológica y 13\% no oncológica; y úlceras por presión de III o IV grado en $5 \%$ de los oncológicos y $17 \%$ de los no oncológicos, siendo sus localizaciones más frecuentes sacra, talones y trocánteres.
No oncológicos

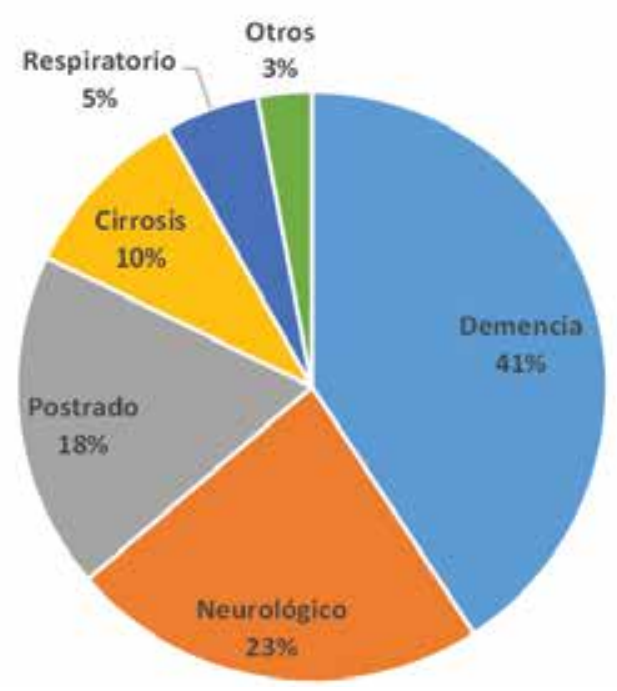

Figura 1. Tipo de enfermedad en fase terminal en pacientes admitidos a emergencia de un hospital terciario.

Se identificó tiempo de enfermedad crónica y tiempo de enfermedad terminal en $46 \%$ de los pacientes oncológicos y $21 \%$ de los no oncológicos, siendo su mediana 05 meses (dos meses en estado terminal) y 02 años (un año en fase terminal) respectivamente $(p=0,03)$. Inicialmente todos los pacientes fueron admitidos en salas de observación común de emergencia, pero en el transcurso de su hospitalización 02 casos oncológicos $(5,4 \%)$ y 11 no oncológicos $(8,1 \%)$ pasaron a unidad de cuidados intermedios de emergencia, uno de los cuales permaneció en unidad de cuidados intensivos 34 días donde finalmente falleció.

Los procedimientos invasivos identificados se presentan en la Tabla 2. Se identificó la realización de un procedimiento invasivo probablemente innecesario en 37 pacientes con enfermedad crónica en fase terminal (21,5\%), correspondiendo a $13,5 \%$ de los casos oncológicos y $23,7 \%$ de pacientes no oncológicos. El destino de los pacientes con procedimientos probablemente innecesarios fue alta $11 \%$, mortuorio 35\% y hospitalización 54\%.

La figura 2, muestra el destino de los pacientes al egreso de la emergencia $(p=0,43)$, encontramos un promedio de estancia en emergencia de 8,46 (+/$5,7)$ días en oncológicos y $8,3(+/-5,8)$ días en no oncológicos; mediana 8 y 7 días respectivamente (rango intercuartil 7) $(p=0,90)$. 
Tabla 2. Procedimientos invasivos realizados en pacientes con enfermedad crónica en fase terminal admitidos a emergencia de un hospital terciario.

\begin{tabular}{lccc}
\hline \multicolumn{1}{c}{ Procedimiento } & Oncológicos (37) & $\begin{array}{c}\text { No Oncológicos } \\
\mathbf{( 1 3 5 )}\end{array}$ & Total (172) \\
\hline Probablemente innecesario & $2(5,4 \%)$ & $18(13,3 \%)$ & $20(11,6 \%)$ \\
Intubación orotraqueal & $2(5,4 \%)$ & $18(13,3 \%)$ & $20(11,6 \%)$ \\
Inotrópicos por CVC \& & $1(2,7 \%)$ & $6(4,4 \%)$ & $7(4,1 \%)$ \\
Hemodiálisis & 0 & $2(1,5 \%)$ & $2(1,2 \%)$ \\
Ventilación mecánica invasiva & $37(100 \%)$ & $135(100 \%)$ & $172(100 \%)$ \\
Otros procedimientos & $5(13,5 \%)$ & $66(48,9 \%)$ & $71(41,0 \%)$ \\
Catéter venoso periférico & $3(8,1 \%)$ & $34(25,2 \%)$ & $37(21,5 \%)$ \\
Sonda nasogástrica & $1(2,7 \%)$ & $2(1,5 \%)$ & $3(1,7 \%)$ \\
Sonda urinaria & $1(2,7 \%)$ & $1(0,7 \%)$ & $2(1,2 \%)$ \\
Paracentesis & 0 & $2(1,5 \%)$ & $2(1,2 \%)$ \\
Drenaje torácico & $3(8,1 \%)$ & $1(0,7 \%)$ & $4(2,3 \%)$ \\
\hline Traqueostomía & & & \\
Otros & &
\end{tabular}

\&CVC: catéter venoso central.
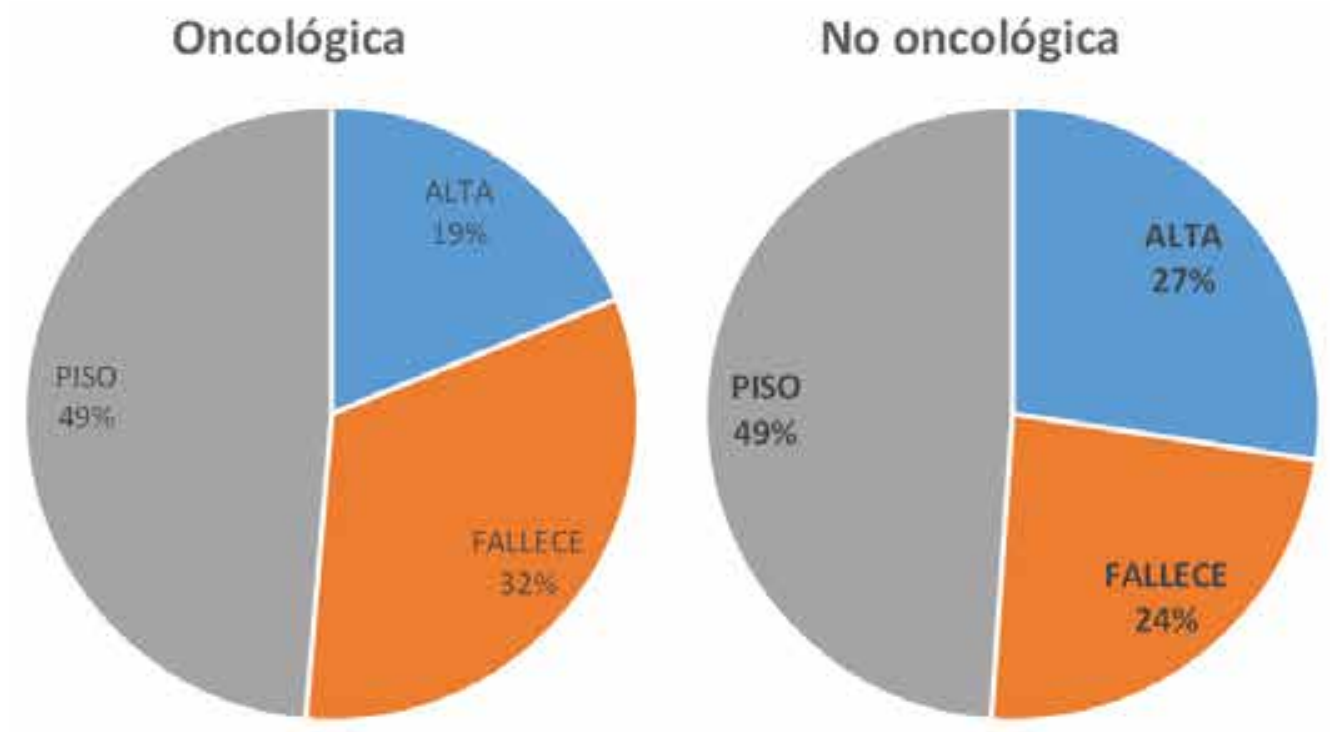

Figura 2. Destino de pacientes con enfermedad crónica en fase terminal admitidos a emergencia de un hospital terciario. 


\section{DISCUSIÓN}

Pacientes con enfermedades crónicas en fase terminal acuden con frecuencia al servicio de emergencia de hospitales de tercer nivel $\left.\right|^{4,14,18,25}$, un porcentaje importante $(41 \%)$ lo hizo por la unidad de shock trauma, área destinada a mantener la vida del paciente mediante procedimientos invasivos. Una proporción considerable (8\%) era enviado a una sala de cuidados críticos (cuidados intermedios), similar a lo reportado en Australia, con resultados desfavorables ${ }^{23}$.

Los síntomas encontrados fueron similares a otros estudios26. Presentando alta frecuencia de úlceras por presión y caquexia, similar a otras publicaciones. Estos pacientes tenían una estancia prolongada en emergencia (ocho días), mayor a la estancia general y los estándares recomendados ${ }^{2}$, debido probablemente a deficiencias de todo el sistema de salud. Casi la mitad de estos pacientes fueron derivados a otros servicios de hospitalización, donde también permanecen un tiempo prolongado y la mayoría fallece ${ }^{18}$

El servicio de emergencia es una oportunidad para identificar a este tipo de pacientes e incluirlos en programas de cuidados paliativos (ambulatorios o domiciliarios $)^{16}$. Aunque en nuestro medio la oferta de cuidados paliativos es inadecuada e insuficiente, esta intervención disminuyó significativamente la estancia en emergencia y el ingreso a otros servicios del hospital, incluyendo unidades críticas.

Se encontró un porcentaje importante de procedimientos invasivos considerados innecesario lo que prolongaría el sufrimiento del paciente y es considerado inadecuado en la calidad de atención del servicio de salud. Al respecto hay escasas publicaciones en nuestro medio, por falta de capacitación del personal y desconocimiento de la familia, que muchas veces exige dichos procedimientos con la intención errada de ayudar al paciente ${ }^{27}$. Si consideramos el enfoque de los cuidados paliativos, otros procedimientos invasivos menores (vía endovenosa, sonda nasogástrica o vesical), tampoco se debería realizar en pacientes en situación al final de la vida ${ }^{5,9,15}$.

Se debería evitar el ingreso de pacientes con enfermedad terminal a la unidad de trauma shock, pues procedimientos invasivos innecesarios pueden incrementar el sufrimiento del paciente y la familia. Para esto se necesita una mejor comunicación, más que hacer el diagnóstico o determinar el pronóstico en ese momento es detectar el antecedente y la expectativa de vida que muchas veces ya se ha definido previamente.
Es necesario personal capacitado o consultar a un equipo de cuidados paliativos, antes de decidir medidas invasivas de soporte vital en pacientes con antecedentes bien definidos. Sin embargo, es complejo, desde el punto de vista ético y en el país no existen bases legales claras. Esta intervención permitiría que ambientes de áreas críticas estén disponibles para pacientes con otras patologías y necesidades.

Las enfermedades no oncológicas, como insuficiencia orgánica o secuelas neurodegenerativas, fueron más frecuentes, similar a lo descrito en otros países, sin embargo, son menos estudiadas que los casos oncológicos, a pesar de presentar mayor sobrevida y uso de servicios de salud ${ }^{21,28,29}$.

La investigación se realizó en un solo hospital con hacinamiento de pacientes, durante un corto periodo de intervención, perteneciente a la seguridad social (que incluye aproximadamente al $40 \%$ de la población nacional). No existe legislación clara en nuestro medio respecto a decisiones al final de la vida y cuidados paliativos, existiendo dudas morales en la toma de decisiones. Algunas veces la información brindada era incompleta o contradictoria. Los conocimientos del paciente, familia y personal de salud respecto a criterios de terminalidad en enfermedades crónicas, no son claros, sobre todo en temas relacionados a la muerte.

\section{CONCLUSIÓN}

En conclusión, el $10 \%$ de los pacientes admitidos a emergencia de un hospital terciario presentan enfermedad crónica terminal, con mayor frecuencia no oncológica, realizándose procedimientos probablemente innecesarios en la quinta parte de estos, con estancia prolongada y alta tasa de fallecimiento.

Se recomienda mejorar la captación de pacientes con enfermedad crónica en fase terminal, aprovechando la oportunidad de su asistencia a servicios de emergencia de hospitales terciarios y coordinar con los servicios de cuidados paliativos para su seguimiento y control. Evaluando la pertinencia de procedimientos invasivos en estos pacientes que podría prolongar el sufrimiento y tener presente la necesidad de administrarles cuidados paliativos. Capacitar al personal de salud del servicio de emergencia en estos temas o implementar un sistema de atención especial en estos pacientes, integrado a la unidad de cuidados paliativos. A nivel regional y nacional es necesario impulsar el enfoque paliativo para las enfermedades crónicas avanzadas, oncológicas y no oncológicas. 
Contribuciones de autoría: José Amado-Tineo: a,b,c,d,f,g; Waldo Taype-Huamaní: a,b,d,e,g; Rolando Vasquez-Alva: b,d,e,g; Fiorella Siccha-Del Campo: b,d,e,g;Teodoro Oscanoa-Espinoza: a,b,c,d,e,g

(a. Concepción y diseño del trabajo; b. Recolección / obtención de datos; c. Contribución estadística; d. Análisis e interpretación de datos; e. Revisión crítica del manuscrito; f. Redacción del manuscrito; g. Aprobación de su versión final).

Correspondencia: José Percy Amado Tineo.

Dirección: Jr. Belisario Flores 238 Dpto 301, Lince, Lima - Perú.

Teléfono: +1990452547

Correo:jpamadot@gmail.com;jpamadot@hotmail.com
Financiamiento: Premio Kaelin en ciencias de la salud - Protocolos de Investigación 2016. Instituto de Evaluación de Tecnologías en Salud e Investigación (IETSI) EsSalud.

Conflicto de interés: Los autores declaran no tener conflicto de interés en la publicación de este artículo.

Recibido: 06 de abril 2019

Aprobado: 16 de mayo 2019

\section{REFERENCIAS BIBLIOGRÁFICAS}

1. Ministerio de salud. Norma Técnica de los Servicios de Emergencia de Hospitales del Sector Salud [Internet]. Lima-Perú; 2004. Available from: http://bvs.minsa.gob.pe/local/dgsp/NT042emerg.pdf

2. Vásquez Alva R, Amado Tineo J, Ramírez Calderón F, Velásquez Velásquez R, Huari Pastrana R. Sobredemanda de atención médica en el servicio de emergencia de adultos de un hospital terciario, Lima, Perú. An la Fac Med 2016;77(4):379. Available from: http://www.scielo.org.pe/ scielo.php?script=sci_arttext\&pid=S102555832016000400010

3. Amado J, Vasquez R, Huari R, Palacios A. Readmision frecuente de pacientes al servicio de emergencia de adultos de un hospital terciario. Lima-Peru 2012. Rev Soc Peru Med Interna. 2015;28(4):153-7.

4. George N, Phillips E, Zaurova M, Song C, Lamba S, Grudzen C. Palliative Care Screening and Assessment in the Emergency Department: A Systematic Review. J Pain Symptom Manage. 2016;51(1):108-119. e2. Available from: http://linkinghub.elsevier.com/retrieve/pii/ S0885392415004443

5. World Health Organization (WHO). Global Atlas of Palliative Care at the End of Life. Geneva, Switzerland.; 2014. 111 p.

6. Da Silva Soares D, Nunes CM, Gomes B. Effectiveness of Emergency Department Based Palliative Care for Adults with Advanced Disease: A Systematic Review. J Palliat Med. 2016;19(6):601-9. Available from: http://online.liebertpub.com/doi/10.1089/jpm.2015.0369

7. Quiñones Pérez A, Álvarez Jiménez P, García Sánchez MJ, Guardia Mancilla P. Influencia de la atención avanzada en cuidados paliativos en la frecuentación de las urgencias hospitalarias. Med Paliativa. 2013;20(2):60-3. Available from: http://linkinghub.elsevier.com/ retrieve/pii/S1134248X12000791

8. Van Tricht $M$, Riochet $D$, Batard $E$, Martinage $A$, Montassier $E$, Potel $G$, et al. Palliative care for patients who died in emergency departments: analysis of a multicentre cross-sectional survey. Emerg Med J. 2012;29(10):795-7. Available from: http://emj.bmj.com/lookup/ doi/10.1136/emermed-2011-200513

9. Lafond P, Chalayer E, Roussier M, Weber E, Lacoin-Reynaud Q, Tardy B. A Hospice and Palliative Care Bed Dedicated to Patients Admitted to the Emergency Department for End-of-Life Care. Am J Hosp Palliat Med. 2016;33(4):403-6. Available from: http://ajh.sagepub.com/cgi/ doi/10.1177/1049909114562947

10. Ali AA, Adam R, Taylor D, Murchie P. Use of a structured palliative care summary in patients with established cancer is associated with reduced hospital admissions by out-of-hours general practitioners in Grampian.

\section{BMJ Support Palliat Care. 2013 Dec;3(4):452-5.}

11. Perkins P, Booth S, Vowler SL, Barclay S. What are patients' priorities for palliative care research? -- a questionnaire study. Palliat Med 2008;22(1):7-12. Available from: https://www.ncbi.nlm.nih.gov/ pubmed/18216073

12. Sharma N, Sharma AM, Wojtowycz MA, Wang D, Gajra A. Utilization of palliative care and acute care services in older adults with advanced cancer. J Geriatr Oncol 2016;7(1):39-46. Available from: http:// linkinghub.elsevier.com/retrieve/pii/S1879406815003240

13. Wang L, Piet L, Kenworthy CM, Dy SM. Association between palliative case management and utilization of inpatient, intensive care unit, emergency department, and hospice in Medicaid beneficiaries. Am J Hosp Palliat Care 2015;32(2):216-20. Available from: http://ajh. sagepub.com/cgi/doi/10.1177/1049909113520067

14. Wong J, Gott M, Frey R, Jull A. What is the incidence of patients with palliative care needs presenting to the Emergency Department? A critical review. Palliat Med 2014;28(10):1197-205. Available from: http:// journals.sagepub.com/doi/10.1177/0269216314543318

15. Metola Gómez A, García Romo E, Bellido De Vega S, Carretero Lanchas Y, Azcoitia Álvarez B, Guerrero L. Análisis de la evolución de pacientes en probable situación de últimos días en un servicio de Urgencias. Med Paliativa. 2013;20(1):10-8.

16. Liberman $T$, Kozikowski A, Kwon N, Emmert B, Akerman $M_{\text {, }}$ Pekmezaris R. Identifying Advanced Illness Patients in the Emergency Department and Having Goals-of-Care Discussions to Assist with Early Hospice Referral. J Emerg Med 2018;54(2):191-7. Available from: http:// linkinghub.elsevier.com/retrieve/pii/S073646791730714X

17. Amado J. Necesidad y demanda de cuidados paliativos en pacientes readmitidos a emergencia de adultos de hospitales nacionales del seguro social, Lima 2016. Universidad Nacional Mayor de San Marcos; 2017.

18. Amado JP, Vasquez R, Huari RW, Sucari A, Oscanoa T. Patients with End-stage Oncologic and Nononcologic Disease in Emergency Service of an Urban Tertiary Hospital. Indian J Palliat Care 2018;24(1):25-7. Available from: https://www.ncbi.nlm.nih.gov/pubmed/29440802

19. Smith AK, McCarthy E, Weber E, Cenzer IS, Boscardin J, Fisher J, et al. Half Of Older Americans Seen In Emergency Department In Last Month Of Life; Most Admitted To Hospital, And Many Die There. Health Aff 2012;31(6):1277-85. Available from: http://www.ncbi.nlm.nih.gov/ pmc/articles/PMC3736978/?tool=pubmed

20. Lawson BJ, Burge Fl, Mcintyre P, Field S, Maxwell D. Palliative care patients in the emergency department. J Palliat Care. 2008;24(4):247-55. 
21. Glajchen $M$, Lawson $R$, Homel $P$, Desandre $P$, Todd $K H$. A rapid two-stage screening protocol for palliative care in the emergency department: a quality improvement initiative. J Pain Symptom Manage 2011;42(5):657-62. Available from: http://linkinghub.elsevier.com/ retrieve/pii/S0885392411004039

22. Ouchi K, Wu M, Medairos R, Grudzen CR, Balsells H, Marcus D, et al. Initiating Palliative Care Consults for Advanced Dementia Patients in the Emergency Department. J Palliat Med 2014;17(3):346-50. Available from: http://online.liebertpub.com/doi/abs/10.1089/jpm.2013.0285

23. Sulistio M, Franco M, Vo A, Poon P, William L. Hospital rapid response team and patients with life-limiting illness: A multicentre retrospective cohort study. Palliat Med. 2015;29(4):302-9. Available from: http:// journals.sagepub.com/doi/10.1177/0269216314560802

24. Fachado AA, Martínez NS, Roselló MM, Ria JJV, Oliver EB, García RG, et al. Spanish adaptation and validation of the supportive \&amp; palliative care indicators tool - SPICT-ESTM. Rev Saude Publica 2018;52:3. Available from: http://www.revistas.usp.br/rsp/article/view/142383

25. Le Conte P, Riochet D, Batard E, Volteau C, Giraudeau B, Arnaudet I, et al. Death in emergency departments: a multicenter cross-sectional survey with analysis of withholding and withdrawing life support.
Intensive Care Med 2010;36(5):765-72. Available from: http://dx.doi. org/10.1007/s00134-010-1800-1

26. Alsirafy SA, Raheem AA, Al-Zahrani AS, Mohammed AA, Sherisher MA, El-Kashif AT, et al. Emergency Department Visits at the End of Life of Patients With Terminal Cancer: Pattern, Causes, and Avoidability. Am J Hosp Palliat Med 2016;33(7):658-62. Available from: http://ajh. sagepub.com/cgi/doi/10.1177/1049909115581819

27. Australasian College for Emergency Medicine. Policy on end of life and palliative care in the emergency department (P455). Emerg Med Australas 2016;28(5):617-21. Available from: http://doi.wiley. com/10.1111/1742-6723.12674

28. Aira Ferrer P, Domínguez Lorenzo C, Cano González JM, García Verde I, Molina Baltanás E, Vicente Aedo M, et al. Características de los pacientes con enfermedad crónica avanzada incluidos en un programa de cuidados paliativos domiciliario. Med Paliativa 2015; Available from: http://linkinghub.elsevier.com/retrieve/pii/S1134248X15000841

29. Rosenwax LK, McNamara BA, Murray K, McCabe RJ, Aoun SM, Currow DC. Hospital and emergency department use in the last year of life: a baseline for future modifications to end-of-life care. Med J Aust. 2011 Jun;194(11):570-3.

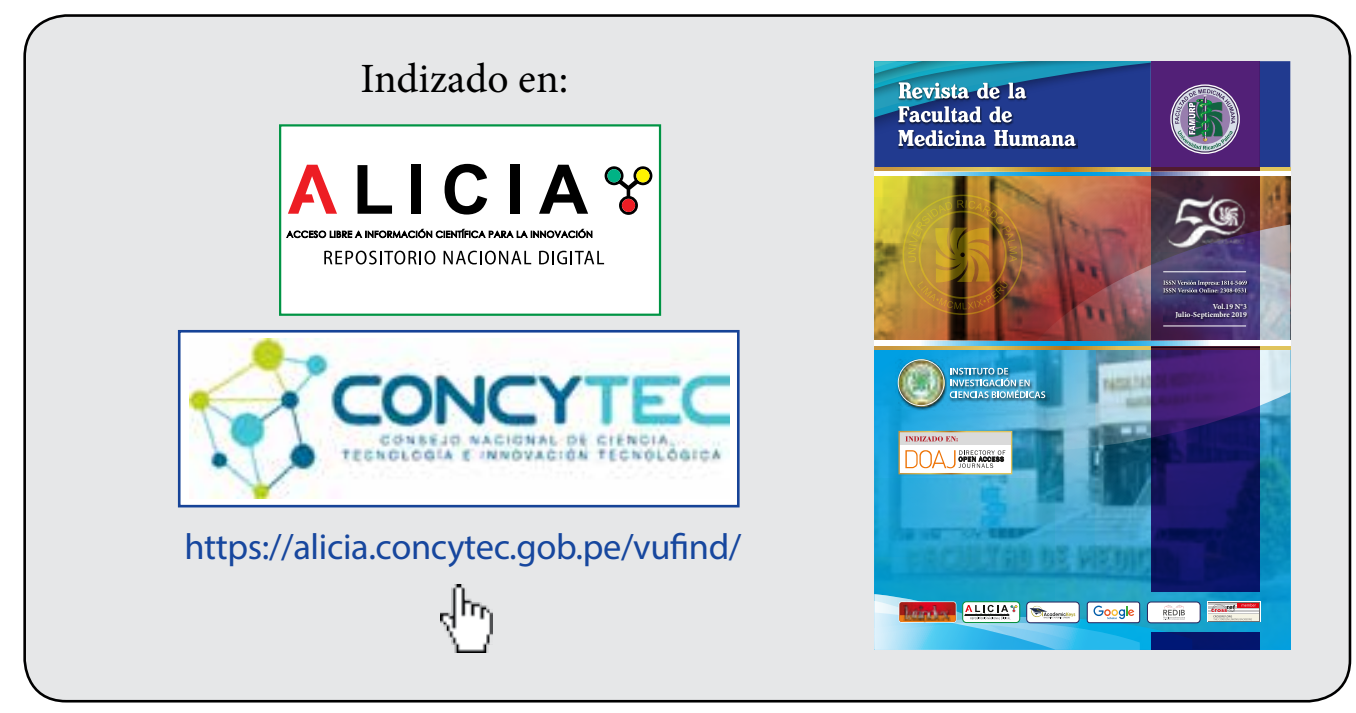

